\section{Too much of a good thing}

After being activated, microglia in the central nervous system (CNS) fail to acquire an anti-inflammatory phenotype, and bone marrow-derived monocytes must be recruited for resolution of the microglia-induced inflammation. In The EMBO Journal, Cohen et al. show that extended exposure to the cytokine TGF- $\beta$ impairs the conversion of proinflammatory (M1) macrophages into anti-inflammatory (M2) macrophages by dampening the expression of the transcription factor IRF7. Preexposure to TGF- $\beta$, which is highly expressed in the CNS, prevents induction of the M2 phenotype during the late-stage response to lipopolysaccharide in microglia or bone marrow-derived macrophages and downregulates expression of interleukin 10 (IL-10) and the transcription factor IRF7, a profile that is also found ex vivo in microglia isolated from the CNS. Stimulation with interferon- $\beta$, a known inducer of IRF7, allows M1-to-M2 conversion in TGF- $\beta$-exposed microglia in vitro and in vivo. Thus, tissue-specific environmental factors have an essential role in the fate of resident macrophages.

EMBO J. (13 November 2014) doi:10.15252/embj.201489293

\section{Signals for HSC emergence}

In vertebrates, hematopoietic stem cells (HSCs) arise from the hemogenic endothelium in the dorsal aorta during a brief developmental window. In Cell, Traver and colleagues show that signaling by tumornecrosis factor (TNF) through its receptor TNFR2 is required for the emergence of HSCs from the aortic endothelium in zebrafish embryos, but signaling via its receptor TNFR1 is not. TNF signaling induces expression of the Notch ligand Jagged 1 to activate Notch1 in the hemogenic endothelium and also activates the transcription factor NF- $\kappa \mathrm{B}$. Both Notch 1 and NF- $\kappa \mathrm{B}$ are required for HSC specification. These effects are independent of the role of TNF in the developing vasculature or the induction of apoptosis in endothelial cells. Yolk sack-derived primitive neutrophils are the main source of TNF for the induction of HSCs, which indicates a role for these transient immune cells in definitive hematopoiesis.

Cell 159, 1070-1085 (2014)

\section{Drivers of $B_{\text {reg }}$ cells}

Regulatory B cells ( $B_{\text {reg }}$ cells) help to prevent excessive tissue damage during inflammation by the production of IL-10, but the drivers of $B_{\text {reg }}$ cell generation have yet to be delineated. In Nature Medicine, Rosser et al. show that gut microbiota contribute to the differentiation of splenic $B$ cells into $B_{\text {reg }}$ cells. Antibiotictreated mice have fewer IL-10-producing $B_{\text {reg }}$ cells. In vitro stimulation with antibody to the costimulatory receptor CD40, IL- 6 or IL-1 $\beta$, alone or in combination, induces B cell proliferation and IL-10 production. Chimeric mice lacking B cell expression of the cytokine receptors IL-6R or IL-1R1 have not only fewer $B_{\text {reg }}$ cells but also defects in suppressing inflammatory arthritic disease. These findings suggest that gut microbes induce a proinflammatory response that feeds back onto splenic $B$ cells to induce their conversion to the $\mathrm{B}_{\text {reg }}$ cell phenotype. LAD Nat. Med. 20, 1334-1339 (2014)

\section{A nonspecific boost}

Flagellin is the main microbe-associated motif recognized in the gut, and this is mediated by the host sensor molecules TLR5 and NLRC4. In Science, Gewirtz and colleagues harness the flagellin-recognition pathway to enhance resistance in a mouse model of rotavirus infection. The administration of flagellin before a high infectious dose of rotavirus results in considerable resistance to infection even in RAG recombinase-deficient mice, which completely lack adaptive immunity, or mice that lack interferon signaling. This resistance is mediated instead by dendritic cells that produce the proinflammatory cytokines IL-12, IL-18 and IL-23 in response to flagellin. These cytokines have two main effects: first, to drive the activation of innate lymphoid cells, and second, to induce the apoptosis of intestinal epithelial cells, which robs rotavirus of its reproductive niche. Activated innate lymphoid cells in turn release IL-22, which elicits an antiviral gene-expression program in intestinal epithelial cells. Stimulation of innate immunity may therefore be potentially effective in diverse infections.

Science 346, 861-865 (2014)

\section{Distinct tumor APCs}

Tumor environments contain immunosuppressive antigenpresenting cells (APCs) that can prevent eradication of the tumor mediated by tumor-specific $\mathrm{CD}^{+} \mathrm{T}$ cells. In Cancer Cell, Broz et al. profile APC subsets in various tumors. Although tumor-associated macrophages are abundant at tumor margins, a rare CD103+ dendritic cell (DC) subset is also present in tumors. The $\mathrm{CD} 103^{+}$population responds to the cytokines GM-CSF and FIt3L. Analogous BDCA3+ DCs are present in human tumors. These $\mathrm{CD}_{103^{+}}\left(\mathrm{BDCA}^{+}\right)$ DCs express IL-12 and cross-present antigen to prime naive and activate effector $\mathrm{CD}^{+} \mathrm{T}$ cells. Ablation of these DCs in mice blunts tumor regression mediated by adoptive $\mathrm{T}$ cell therapy. Notably, retrospective analyses of human tumor geneexpression profiles positively correlate $\mathrm{BDCA}^{+} \mathrm{DC}$ signatures and patient prognosis. These findings suggest that boosting the frequency of this BDCA3+ DC subset might further enhance antitumor immunotherapy. Cancer Cell (10 November 2014) doi:10.1016/j.ccell.2014.09.007

\section{$T_{\text {reg }}$ cells' peripheral helpers}

Lymph node (LN) stromal cells can express major histocompatibility complex (MHC) class II, but the full relevance of this expression has been unclear. In eLife, Mebius and colleagues use LN transplants to demonstrate a key role for stromal cell MHC class II in the maintenance of peripheral immunological homeostasis. MHC class II-deficient LNs transplanted into wild-type hosts accumulate activated T cells, and such transplants are eventually rejected, presumably due to intense activation of the cells that they are housing. This effect is localized to the transplanted MHC class II-deficient LNs, because endogenous wild-type LNs in the same host are intact. Mechanistically, the expression of MHC class II by LN stroma seems to specifically support the homeostatic proliferation of regulatory $\mathrm{T}$ cells via the presentation of antigen. This function seems to be relevant for the maintenance of peripheral tolerance, as LN grafts that express specific antigen can regulate experimental models of delayed-type hypersensitivity responses and rejection of skin grafts. eLife (19 November 2014) doi:10.7554/eLife.04433 